\title{
Charge Transfer in Dimer with Dissipation
}

\author{
Nadezhda Fialko ${ }^{1, \star}$, Maxim Olshevets ${ }^{1}$, and Victor Lakhno ${ }^{1}$ \\ ${ }^{1}$ Institute of Mathematical Problems of Biology, Russian Academy of Sciences, RU-142290, Pushchino, Moscow Region, Russia
}

\begin{abstract}
The study of the charge transfer processes in biomacromolecules such as DNA is essential for the development of nanobioelectronics, design and construction of DNA-based nanowires, memory devices, logical elements, etc. Mathematical and computer modeling of charge transfer in biopolymer chains is an important part of these investigations. Some properties of charge transfer can be demonstrated by modeling of two-site chain. Based on the semi-classical Holstein model we consider a system of two sites and charged particle (electron or hole) in which the oscillations of the first site are not related to the charge motion, and the parameters of the second site correspond to a small-radius polaron. The system steady states depending on the electron energy $\mathrm{H}$ at the second site are studied numerically. The dynamics of the charge initially localized at the first site is modeled. Various modes depending on $\mathrm{H}$ are demonstrated: charge tunneling, resonant transfer, and lack of transfer.
\end{abstract}

\section{Introduction}

Mathematical modeling of charge transfer processes in biological systems is associated with the use of discrete models, in which the paths of charge transfer in macromolecules are considered. To date, many works have been published on modeling the motion of a charged particle in various types of molecular chains [1-3]. One of the first polaron models of charge propagation in a onedimensional chain was proposed by Holstein [4]. He considered a homogeneous chain of molecules (sites) as a chain of harmonic oscillators, under the assumption that the displacement of the site from the equilibrium position affects the charge energy at this site.

Some important properties of the dynamics of selfconsistent quantum-classical systems can be demonstrated by the example of a two-site model. For two identical sites in the Holstein model, the problem was investigated both analytically and by computer simulation ([5-7] and references therein). In quantum dimer, the case of sites with different electron energy was modeled $[8,9]$.

In this work we consider a simple heterogeneous case when displacements of the first site do not interact with a charge. The parameters of the second site correspond to a small-radius polaron (when in a long homogeneous chain the charge is localized on one site with the probability of $\approx 1$ ). In numerical modeling, we took parameter values close to (A/T) DNA fragments. The results of biophysical experiments on charge transfer in DNA suggest the possibility of using DNA in nanobioelectronics $[10,11]$.

\section{Charge transfer model for dimer}

The model is based on the Holstein Hamiltonian for a discrete chain of sites [4], which in dimensionless variables [12] have the form

$$
\begin{aligned}
H= & \sum_{m \neq n} \eta_{n m} b_{m} b_{n}^{*}+\sum_{n} \eta_{n n} b_{n} b_{n}^{*}+ \\
& +\frac{1}{2} \sum_{n} \omega^{2} u_{n}^{2}+\frac{1}{2} \sum_{n} v_{n}^{2}+\sum_{n} \chi_{n} u_{n} b_{n} b_{n}^{*} .
\end{aligned}
$$

Here $b_{n}$ is the amplitude of the probability of the charge occurrence on the $n$th site (complex variable), $u_{n}$ is displacement of the $n$th site from its equilibrium position, $v_{n}$ is the velocity of the $n$th site. Parameters: $\eta_{m n}(m \neq n)$ are matrix elements of the transition between the $m$ th and $n$th sites, $\eta_{n n}$ is the electron energy at the $n$th site, $\omega$ is frequency of site oscillations. Last term taking into account interaction of site displacement $u_{n}$ with charge probability $b_{n} b_{n}^{*}$ on this site, $\chi_{n}$ is the constant of coupling between quantum and classical subsystems. The total probability of finding a charge in the system is $\sum_{n} b_{n} b_{n}^{*}=1$.

Here we consider the system of 2 sites $(n=1,2)$, with "rigid" first site $\left(\chi_{1}=0\right)$, so, the classical displacements of the first site do not affect the dynamics of the charge and can be ignored. Choose electron energy on the first site $\eta_{11}=0$, and denote $\eta_{22}=\mathrm{H}$, matrix element $\eta_{12}=\eta$, $\chi_{2}=\chi$ and $u_{2}=u$. Motion equations for Hamiltonian (1) have the form

$$
\begin{aligned}
& i \frac{d b_{1}}{d t}=\eta b_{2}, \\
& i \frac{d b_{2}}{d t}=\eta b_{1}+\mathrm{H} b_{2}+\chi u b_{2},
\end{aligned}
$$

^e-mail: fialka@impb.ru 


$$
\begin{aligned}
& \frac{d u}{d t}=v, \\
& \frac{d v}{d t}=-\omega^{2} u-\chi\left|b_{2}\right|^{2}-\gamma v .
\end{aligned}
$$

We supplemented eq. (5) with a term with friction ( $\gamma$ is the friction coefficient). In the presence of dissipation in the classical subsystem, the evolution of the system will continue until it reaches one of its stationary states.

\section{Steady states of the system}

There are no equilibrium points in the model, but there are steady states where the site is stationary, $\left|b_{1}\right|$ and $\left|b_{2}\right|$ are constants, and only the phases of variables $b_{1}$ and $b_{2}$ change. The total energy of the system corresponding to the Hamiltonian (1) in this case has the form

$$
E=\eta\left(b_{1} b_{2}^{*}+b_{1}^{*} b_{2}\right)+\mathrm{H} b_{2} b_{2}^{*}+\chi u b_{2} b_{2}^{*}+\frac{\omega^{2}}{2} u^{2}+\frac{1}{2} v^{2} .
$$

Put $b_{n}=r_{n} \exp \left(i \varphi_{n} t\right), \varphi=\left(\varphi_{1}-\varphi_{2}\right)$, and $r_{2}=r$, then $r_{1}=\sqrt{1-r^{2}}$. The energy of the system (6)

$$
E=2 \eta r \sqrt{1-r^{2}} \cos \varphi+(\mathrm{H}+\chi u) r^{2}+\frac{\omega^{2}}{2} u^{2}+\frac{1}{2} v^{2} .
$$

Necessary conditions for steady states

$$
\begin{aligned}
\frac{\partial E}{\partial u}=0 \quad \Rightarrow \quad \omega^{2} u+\chi r^{2}=0 \quad \Rightarrow \quad u=-\frac{\chi}{\omega^{2}} r^{2} \\
\frac{\partial E}{\partial v}=0 \quad \Rightarrow \quad v=0 \\
\frac{\partial E}{\partial \varphi}=0 \Rightarrow \varphi=\pi k \\
\frac{\partial E}{\partial r}=0 \quad \Rightarrow \quad \text { (with (8) and (10)) } \\
\quad \pm \frac{2 \eta}{\sqrt{1-r^{2}}}\left(1-2 r^{2}\right)+2 \mathrm{H} r-2 \frac{\chi^{2}}{\omega^{2}} r^{3}=0,
\end{aligned}
$$

so when $\eta>0$ necessary condition for the steady state

$$
F=-\eta \frac{1-2 r^{2}}{\sqrt{1-r^{2}}}+\mathrm{H} r-\frac{\chi^{2}}{\omega^{2}} r^{3}=0
$$

The solution $F(r)=0(12)$ is very difficult to find analytically, but if the parameter values are known for physical reasons, then numerical estimates can be made. We take the values $\chi / \omega=2 ., \eta=0.5$ close to the parameters of the base pair of DNA [12,13] and plot the graphs $y=F(r)$ for different values of $\mathrm{H}$ (Fig. 1), as well as the function

$$
E_{0}=-2 \eta r \sqrt{1-r^{2}}+\mathrm{H} r^{2}-\frac{1}{2} \frac{\chi^{2}}{\omega^{2}} r^{4}
$$

which is obtained from (7) by substituting (8)-(10). If $F(r)=0$, then in the neighborhood of $r$ will be $E_{0} \approx E$ and it is possible to compare the energy values in different stationary states.

For $\mathrm{H}=0$ (the electron energy is the same at both sites) $F(r)=0$ has one solution $r \approx 0.99$ (Fig. 1, the black

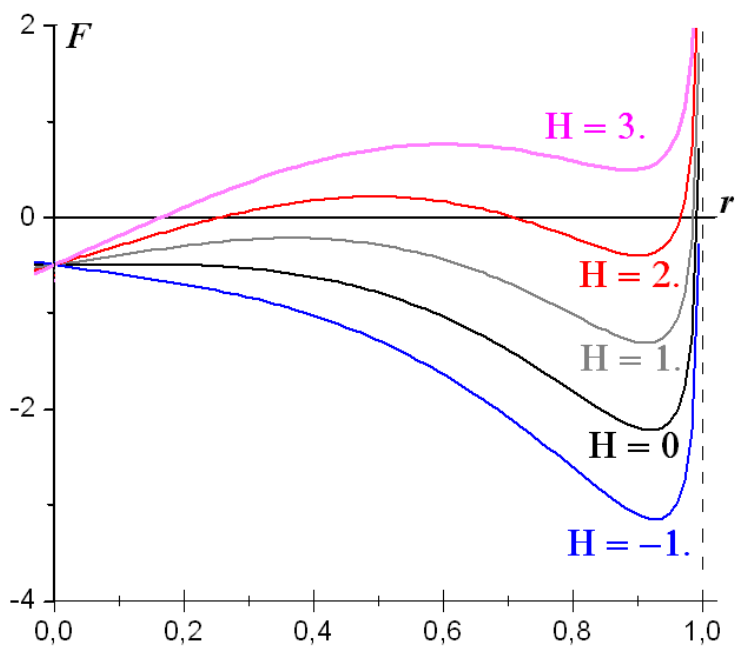

Figure 1. $y=F(r)$ plots for various values of the electron energy $\mathrm{H}$ at the second site.

curve), i.e. if the charge at the initial moment occurs at the first site, then in dynamics the system (2)-(5) will come to a state when the charge with the probability $p=r^{2} \approx 0.98$ is localized at the second site. For negative $\mathrm{H}$ the picture is similar - one solution of $F(r)=0$ at $r \approx 1$ (Fig. 1, blue curve). From a physical point of view, the result is trivial: it is profitable for the charge to be localized at the site where the electron energy is lower.

As $\mathrm{H}>0$ increases to $\approx 1.55$ (with this value of $F(r$ ) it touches the abscissa axis at the point $r \approx 0.45$ ), the picture does not change - the system has one stationary mode, when the charge with the probability $\approx 1$ is localized at the second site (Fig. 1, gray curve). Here, the system demonstrates a simple polaron effect: although the electron energy at the first site $(=0)$ is lower than at the second $(\mathrm{H})$, but due to the interaction with the classical displacement $u$, the minimum of the total energy $E$ (6) will be reached when the charge is localized at the second site.

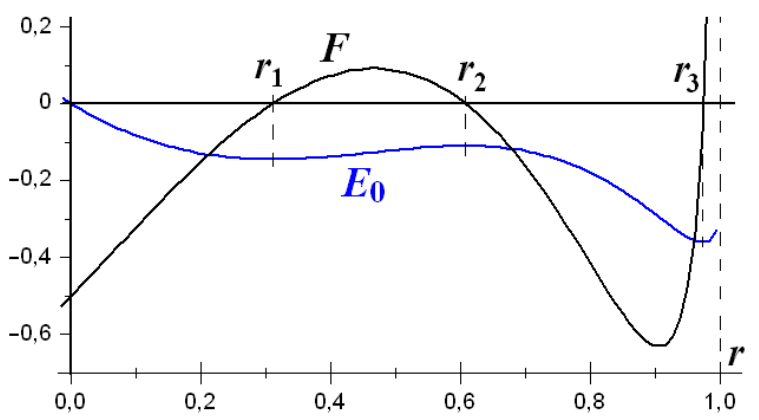

Figure 2. $y=F(r)$ (black curve) and $y=E_{0}(r)$ (blue curve), $\mathrm{H}=1.75$.

With a further increase in $\mathrm{H}$, equation $F(r)=0$ has three solutions (Fig. 1, the red line for $\mathrm{H}=2$ ). Figure 2 shows the plots $F(r)$ and $E_{0}(r)$ for $\mathrm{H}=1.75$ as a detailed example. Calculations of the dynamics of system (2)-(5) from various initial data and with different friction $\gamma$ sug- 


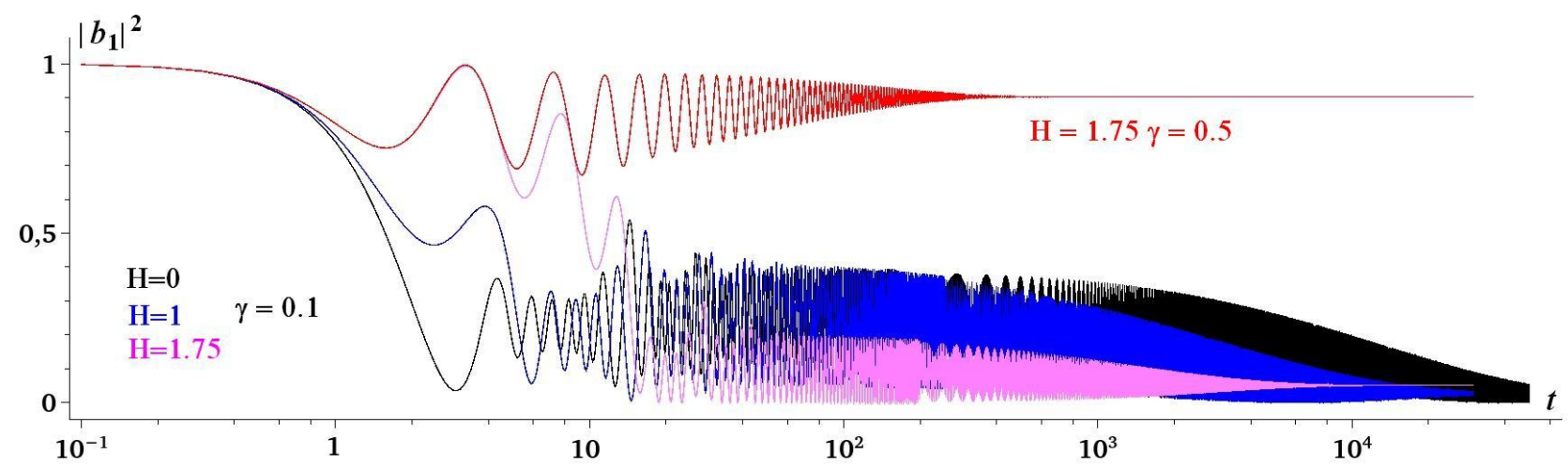

Figure 3. The probability of finding a charge on the first site as a function of time for different values of the electron energy $\mathrm{H} \geq 0$. Graphs are on a semi-logarithmic scale.

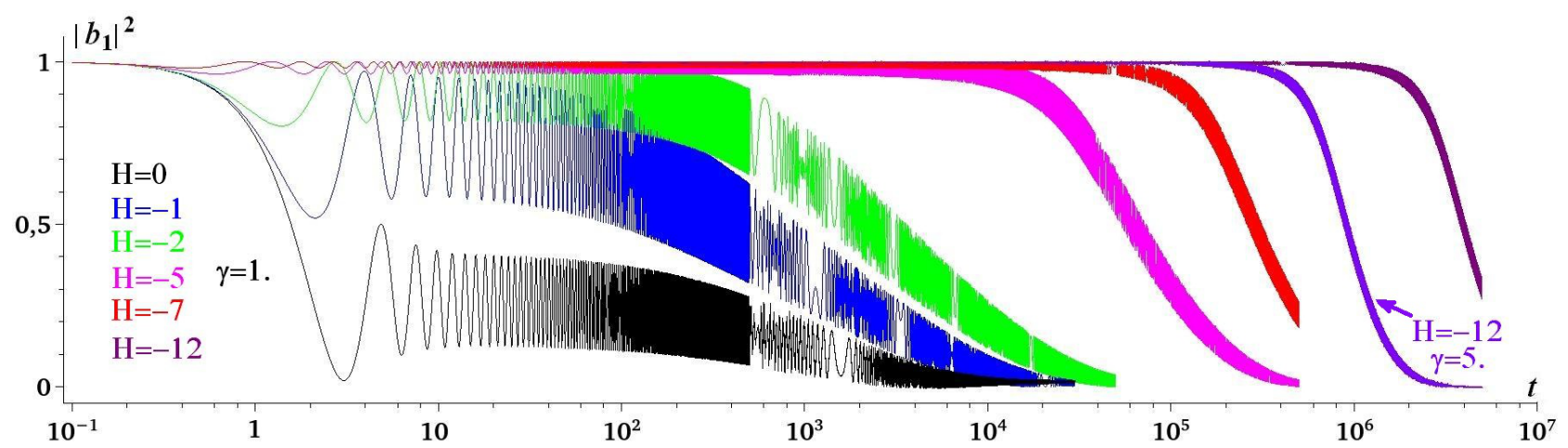

Figure 4. The probability of finding a charge on the first site for different values of the electron energy $\mathrm{H} \leq 0$. For $\mathrm{H}=-12$ the graphs for friction values $\gamma=1$ and $\gamma=5$ (indicated by arrow) are given, for other values of $\mathrm{H} \gamma=1$. Behavior of the graphs for $\mathrm{H}=0,-1$, -2 at $t=500$ is changed due to increasing of the output interval of the results when the trajectory is extended.

gest that the points $r_{1}$ and $r_{3}$ correspond to two minima of the energy $E(6)$. For $\mathrm{H}=2$ the energy is the same at these points, $E\left(r_{1}\right)=E\left(r_{3}\right)=-0.125$. At $\mathrm{H} \approx 2.45 F(r)$ touches the abscissa axis from above, and then there is only one solution for $F(r)=0$ (Fig. 1, pink curve for $\mathrm{H}=3$ ), i.e. the minimal energy corresponds to the state when a charge with the probability of $\approx 1$ is localized at the first site.

\section{Modeling of the charge transfer from the first site}

In a number of experiments on charge transfer in quasione-dimensional biopolymers the charge is localized at one donor site at the initial moment of time. We modeled the dynamics of system (2)-(5) from the initial data $b_{1}(t=0)=1$, the remaining variables are zero. The calculations were performed by the Runge-Kutta fourth-order method. Parameters: $\eta=\omega=0.5, \chi=1$, different values of $\mathrm{H}$ were considered.

For $\mathrm{H} \geq 0$, the transfer takes place in a relatively short time interval. Figure 3 shows the dynamics of the probability of finding a charge on the first site $\left|b_{1}(t)\right|^{2}$ for $\mathrm{H}=0$ and $\mathrm{H}=1$ with friction coefficient $\gamma=0.2 \omega$, and for $\mathrm{H}=1.75$ when $\gamma=0.2 \omega$ and $\gamma=\omega$. At the initial moment $\left|b_{1}(t=0)\right|^{2}=1$, and for a time $t<100$ the probability at the first site becomes less than 0.5 (black, blue, crimson curves), i.e. the charge goes to the second site, and then a long relaxation process follows. The red curve, calculated for the same parameters as the crimson one $(\mathrm{H}=1.75)$ but with larger friction $\gamma=0.5$, indicates that the charge remains at the primary site, i.e. the system fell into the first local minimum (see Fig. 2).

For $\mathrm{H}<0$, the charge transfer occurs over a long time, and the larger $|\mathrm{H}|$, the longer the time interval. Figure 4 shows the results of calculations for different values of $\mathrm{H}$ with friction $\gamma=2 \omega$ and for $\mathrm{H}=-12$ with $\gamma=10 \omega$.

In the system (2)-(5) the site can be considered as a harmonic oscillator with friction $\gamma$ and external force $\left(-\chi\left|b_{2}\right|^{2}\right)$. For a simple oscillator, the decay time is minimal at $\gamma=2 \omega$. In a more complex system (2)-(5)) this is not the case, an increase in friction leads to a decrease in the time $t_{\text {trans }}$ of the charge transfer from the first to the second site (Fig. 4), i.e. to reducing the relaxation time.

For $\mathrm{H}<0$, the transfer time $t_{\text {trans }}$ is exponentially increased with an increase of $|\mathrm{H}|$. For the values of parameters considered $t_{\text {trans }} \sim \exp (4 \sqrt{-\mathrm{H}})$. From a physical point of view, this is a charge tunneling. At $\mathrm{H}>0$, a resonant transfer stage is observed in the dynamics, when the total charge energy at the second site $(\mathrm{H}+\chi u)$ is close to the electron energy of the first site. Then a long relaxation process follows. 
In conclusion, it should be noted that this or similar pattern is typical for a site with parameters of a smallradius polaron: $\eta \ll(\chi / \omega)^{2}$. For set of parameters that describe large-radius polaron it needs more than two sites to obtain such dynamics.

\section{References}

[1] G.B. Schuster (ed.), Long-Range Charge Transfer in DNA II, Topics in Current Chemistry 237 (Springer, 2004)

[2] E.B. Starikov, S. Tanaka, and J.P. Lewis (eds.), Modern Methods for Theoretical Physical Chemistry of Biopolymers (Elsevier Scientific, Amsterdam, 2006)

[3] L. Cruzeiro, J. Biol. Phys. 35, 43 (2009)

[4] T. Holstein, Annals of Physics 8, 325 (1959)
[5] V.M. Kenkre, D.K. Campbell, Phys. Rev. B 34, 4959(R) (1986)

[6] S. Gonçalves, C. Fusco, A.R. Bishop, V.M. Kenkre, Phys. Rev. B 72, 195418 (2005)

[7] M. Tiwari, D.V. Seletskiy, V.M. Kenkre, Eur. Phys. J. B 81, 147 (2011)

[8] C.A. Bustamante, M.I. Molina, Int. J. Mod. Phys. B 17, 2281 (2003)

[9] M. Tiwari, V.M. Kenkre, Eur. Phys. J. B 87, 86 (2014)

[10] V.D. Lakhno, Int. J. Quantum Chem. 108, 1970 (2008)

[11] M. Ratner, Nature Nanotech. 8, 378 (2013)

[12] V.D. Lakhno, N.S. Fialko, Russ. J. Phys. Chem. A 86, 832 (2012)

[13] J. Jortner, M. Bixon, A. A. Voityuk, N. Rösch, J. Phys. Chem. A 106, 7599 (2002) 